Die Misteltherapie an der Schnittstelle zur konventionellen Therapie

Standardisierte Mistelgesamtextrakte stehen unter den pharmakologischen Maßnahmen in der Onkologie seit mehreren Jahrzehnten in der Anwendungshäufigkeit ganz oben. Seit dieser Zeit werden viele positive Erfahrungen von Krebspatienten und von behandelnden Ärzten berichtet. Dennoch besteht ein erheblicher Aufklärungsbedarf, da die Wirksamkeit der Therapie trotz Studiennachweisen auf hohem wissenschaftlichem Niveau (Evidence Based Medicine; EBM) zuweilen kontrovers diskutiert wird. Ganz zu unrecht, wie unlängst eine von dem renommierten Kieler $\mathrm{H}$. G. Creutzfeldt-Institut initiierte Expertenkonferenz zeigte. Unter der Moderation von Prof. Josef Beuth, Köln, diskutierten Experten aus Klinik und Praxis die Bedeutung der Therapie mit dem Mistelgesamtextrakt HELIXOR ${ }^{\circledR}$ und formulierten ihren Konsens in Form eines Thesenpapiers. Die Mistelpräparate werden in der Tumortherapie ergänzend zur onkologischen Standardtherapie eingesetzt, um die körpereigenen Selbstheilungskräfte zu aktivieren, das Tumorwachstum zu hemmen sowie Strahlen- und Chemotherapie assoziierte Nebenwirkungen zu reduzieren.

Insgesamt ist ein möglichst frühzeitiger Therapiebeginn, d.h. in der Akutphase der Erkrankung, anzustreben. Der behandelnde Arzt beginnt mit einer niedrigen Dosierung und steigert langsam bis zur Erhaltungsdosis. In der adjuvanten Tumortherapie hat sich eine Langzeitbehandlung, in der Regel etwa 5 Jahre, etabliert.

\section{Präambel:}

Die Misteltherapie ist ein wesentlicher Bestandteil der evidenzbasierten Tumortherapie. Im Rahmen von EBM-konformen klinischen Studien (Randomized Controlled Trial; RCT und Kohortenstudien) wurden Wirksamkeit und Verträglichkeit einer Therapie mit dem Mistelgesamtextrakt HELIXOR $^{\circledR}$ überprüft. Die Ergebnisse wurden einem Expertengremium zur Bewertung vorgelegt.

1. Die Therapie mit Mistelgesamtextrakten ist in Deutschland die am häufigsten angewandte pharmakologische Maßnahme in der Onkologie [1].

2. Für Mistelgesamtextrakte konnten proliferationshemmende, anti-tumorale, zytotoxische, Apoptose-induzierende, anti-angiogenetische, immunmodulatorische sowie Knochenmark-protektive Effekte belegt werden [2].

3. Kohortenstudien sind international anerkannte epidemiologische Untersuchungen, die zum Nachweis der Unbedenklichkeit und Wirksamkeit praxisrelevanter medizinischer Maßnahmen «Gold Standard» sind [3, 4].

4. Kohortenstudien sind im Wirksamkeitsnachweis mit randomisierten kontrollierten Studien (RCT) vergleichbar und repräsentieren den EBM Grad IIa/b [5, 6].

5. Für Mistelgesamtextrakte liegen klinische Studien der EBM Grade Ia/b und IIa/b vor, die eine indikationsbezogene onkologische Anwendung in der ärztlichen Praxis legitimieren [2].

6. In einem aktuellen RCT senkte der Mistelgesamtextrakt HELIXOR ${ }^{\circledR}$ deutlich und signifikant die Nebenwirkungen von adjuvanten Chemo- und Strahlentherapien und verbesserte damit die Lebensqualität der behandelten Patienten [7].

7. Auch in einer epidemiologischen Kohortenstudie verbesserte der Mistelgesamtextrakt HELIXOR $^{\circledR}$ die Lebensqualität/Beschwerden von Mammakarzinom-Patientinnen nach der turmordestruktiven Standardtherapie (z.B. Operation, Chemo- und Strahlentherapie) signifikant [8].

8. Mit zunehmender Therapiedauer erhöhte sich die Lebensqualität signifikant. Dabei war der Behandlungserfolg in der Regel unabhängig von der Schwere der Erkrankung [2, 8].

9. Nicht-onkologische Begleiterkrankungen und deren Behandlungen hatten keinen Einfluss auf Wirksamkeit und Unbedenklichkeit der Misteltherapie [8].

10. Die komplementäre Verabreichung des Mis- telgesamtextraktes HELIXOR ${ }^{\circledR}$ zeigte in Good Clinical Practice (GCP)/Good Epidemiological Practice (GEP)-konformen RCTs und Kohortenstudien eine sehr gute Verträglichkeit und eine hohe Patientencompliance $[2,7,8]$.

\section{Resümee}

Die Ergebnisse aktueller EBM-konformer klinischer Studien belegen für HELIXOR ${ }^{\circledR}$ eine Verbesserung der Lebensqualität von Tumorpatienten und die Reduktion Chemo-/Strahlentherapie-assoziierter Nebenwirkungen. Im Sinne eines multimodalen Behandlungsansatzes besitzt die Misteltherapie einen wissenschaftlich gesicherten hohen Stellenwert in der Tumortherapie.

Diese Thesen werden inhaltlich getragen von: Prof. Josef Beuth, Köln; Prof. Ulrich Borchard, Düsseldorf; Prof. Volker Fintelmann, Hamburg; Prof. Wolf-D. Gerber, Kiel; Prof. Wolfgang Giebel, Schönebeck; Dr. med. Harald Matthes, Berlin; Prof. Jörg Schulz, Berlin-Buch; Prof. Gerhard Uhlenbruck, Köln.

Quelle

Expertenkonferenz 20.11.06 Frankfurt a.M. Veranstalter: H. G. Creutzfeldt-Institut, Kiel

\section{Literatur}

1 Unger C, Weiss J: Schattauer Verlag 2005.

2 Kienle G, Kiene H: Schattauer Verlag 2003.

3 Schneider B: Cancer Chemother Pharmacol 47:

35-37, 2001.

4 Feinstein AR: Stat Med 3:341, 1984.

5 Benson K, Hartz AJ: New Engl J Med 342:1878-1886, 2000.

6 Concato J, Shah N, Horwitz RJ: New Engl J Med

342:1887-1892, 2000.

7 Piao et al: Anticancer Res 24, 2004.

8 Beuth et al: Submitted for publication, 2007.

Weitere Informationen bei

Helixor Heilmittel GmbH

Fischermühle 1

72348 Rosenfeld

Tel. +49 7428 935-0

\title{
Ticker+++ Ticker+++ Ticker+++ Ticker+++ Ticker+++ Ticker+++ Ticker+++
}

Karl und Veronica Carstens-Stiftung. Der Vorstand der Carstens-Stiftung hat eine Stiftungsprofessur zur Forschung in der Komplementärmedizin/Integrativen Medizin an der Berliner Charité bewilligt. Der Förderzeitraum beträgt fünf Jahre. Eingerichtet wird die Stelle am Institut für Sozialmedizin, Epidemiologie und Gesundheitsökonomie der Berliner Charité.

Weitere Informationen be

Dr. Henning Albrecht

Geschäftsführer der Carstens-Stiftung

Am Deimelsberg 36, 45276 Essen

Tel. +49 201563 05-0
Cefak KG. Mit der Produktneueinführung von Cefamag $^{\circledR} 300$ zum 1. April 2007 ergänzt Cefak seine Mineralstoffpräparatepalette. Eine Filmtablette enthält $300 \mathrm{mg}$ Magnesium. Der tägliche Bedarf von 300 bis $400 \mathrm{mg}$ (Empfehlung der Deutschen Gesellschaft für Ernährung) kann bei magnesiumarmen Nahrungsmitteln, Sportarten mit intensiver Muskelbeanspruchung u.v.a.m. oftmals nicht gedeckt werden.

Weitere Informationen bei Cefak KG

Beatrix Vogler

Ostbahnhofstraße 15, 87437 Kempten

Tel. +49 831 57401-15
AAZ-Pharma. Das Enzymprodukt WobeMugos $₫$ E von der niederländischen Firma AAZ-Pharma B.V. wird ab sofort als NEOZYM ${ }^{\circledR}$ E angeboten. An der Rezeptur der Enzymkombination ändert sich nichts: Auch das neue Produkt enthält 1.740 F.I.P.-Einheiten hochaktiver Enzyme. Die Produktion von NEOZYM ${ }^{\circledR} E$ erfolgt nach GMP-Richtlinien.

Weitere Informationen bei

AAZ-Pharma B.V.

Hamelandroute $91 \mathrm{c}, \mathrm{NL}-7121 \mathrm{JC}$ Aalten

Tel. +3154347588

Info@aazpharma.nl 


\section{SELF-Deutschland-Award an Pascorbin $^{\circledR}$}

Während einer Festveranstaltung am 31. Januar 2007 verlieh die europäische Liga für ein sicheres Patienten-Therapie-Selbstmanagement, SELF e.V., unter der Moderation von Prof. Dr. V. Fintelmann ihren diesjährigen DeutschlandAward an das hoch dosierte Vitamin-C-Infusionspräparat Pascorbin ${ }^{\circledR}$.

Dieses Arzneimittel verbessere in allen Phasen der Tumorerkrankung Lebensqualität und Immunstatus der Patienten und habe einen günstigen Einfluss auf das Nebenwirkungsprofil der Tumorstandardtherapien wie Operation, Chemo- und Radiotherapie. Weiterhin habe es positive Effekte bei zahlreichen anderen schwerwiegenden Krankheitsbildern, z.B. bei rheumatischen und allergischen Erkrankungen sowie bei kritisch Kranken in der Kardiologie, so die Laudatio.

SELF e.V., eine europaweite Initiative von etwa 2000 Ärzten und Wissenschaftlern, gilt als Forum für ein sicheres Patienten-Therapie-Selbstmanagement. Eines der Kernziele dieser europäischen Liga ist es, eine Informations-, Diskussions- und Bewertungsplattform für medizinische Therapien anzubieten. Hierbei werden nicht nur Maßnahmen zur Compliance-Optimierung untersucht, sondern auch Konzepte für eine selbstbestimmte Behandlung in ihrer therapeutischen Effizienz analysiert. In einer Festveranstaltung mit namhaften Experten wurde unlängst unter der Leitung des SELF-Vizepräsidenten Prof. Dr. Dieter Müller, Hamburg, und unter der Moderation von Prof. Dr. Volker Fintelmann, Hamburg, der SELF-Deutschland-Award 2007 an das hoch dosierte Vitamin-C-Infusionspräparat Pascorbin ${ }^{\circledR}$ auf einstimmigen Jurybeschluss verliehen.

Hohe parenterale Bioverfügbarkeit gewährleistet optimale Ascorbatspiegel

In seinem Festvortrag erläuterte Prof. Dr. Dr. Ulrich Borchard, Düsseldorf, dass Tumorerkran- kungen und ihre Behandlung durch Operation, Chemo- und Radiotherapie Allgemeinzustand und Lebensqualität der Betroffenen ganz erheblich einschränken. Er zeigte sich aus Sicht des klinischen Pharmakologen beeindruckt von den positiven Effekten hoch dosierter Vitamin-C-Infusionen insbesondere bei Tumorerkrankungen. «In Fällen von akuten klinischen Vitamin-CMangelzuständen (z.B. bei Operationen, Tumoren und deren Behandlung), die über die Ernährung nicht behoben werden können, ist es notwendig, parenteral mit Vitamin C im HochdosisBereich (Pascorbin ${ }^{\circledR}$ 7,5 g) zu behandeln, um optimale physiologische Ascorbatspiegel wiederherzustellen. Dies ist durch die vollständige Bioverfügbarkeit bei parenteraler Applikation gewährleistet. Im Akutfall können auf diese Weise sehr schnell reduzierte Plasma- und Gewebespiegel aufgefüllt werden», betonte Borchard. Prof. Dr. Dieter Müller konnte diese Ergebnisse aus eigener neurochirurgischer Erfahrung bestätigen: „In eigenen Beobachtungen an operierten Parkinson-Patienten konnten wir post-operativ durch hoch dosierte Vitamin-C-Infusionen eine deutliche Reduktion der Liegezeiten auf Intensivstationen erreichen." Dies zeigt das hohe Potenzial von Pascorbin ${ }^{\circledR}$ in zahlreichen Indikationen, begründete Müller die SELF-Jury-Entscheidung.

Hoch dosierte Vitamin-C-Infusionen in der Onkologie

Insbesondere bei Tumorerkrankungen konnte die Vitamin-C-Hochdosis-Infusionstherapie einem klaren Benefit auch anhand von wissenschaftlichen Studien belegen. Prof. Dr. Volker Fintelmann berichtete über eine aktuelle klinische Studie an 788 Mamma-Karzinom-Patientinnen, die neben den Tumorstandardtherapien adjuvant eine hoch dosierte Vitamin-C-Infusionstherapie erhielten. Dabei konnte nachgewiesen werden, dass parenteral appliziertes Vitamin C in hohen Dosierungen sowohl lebensqualitätsverbessernde Effekte, z.B. Verbesserung beim Fatigue-Syn-

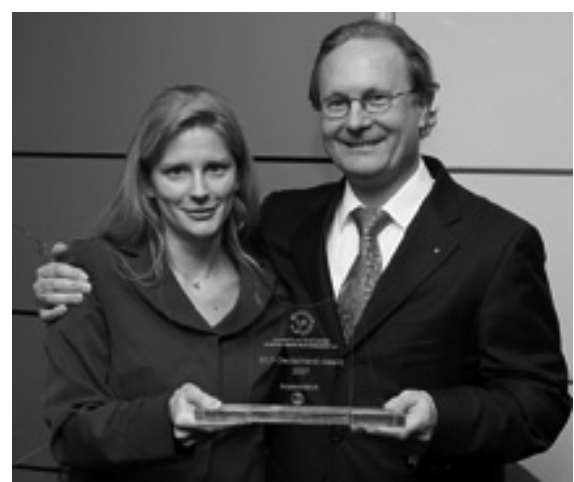

Abb. 1. Annette und Jürgen Pascoe freuen sich über den SELF-Deutschland-Award 2007.

drom, als auch tumorwachstumshemmende Wirkungen aufweist. «Diese Effekte zeigten sich beispielsweise in der Verlängerung des rezidivfreien Intervalls gegenüber der Kontrollgruppe», erklärte Fintelmann.

Insgesamt waren sich die Experten einig, dass die nachweisbar positiven klinischen Wirkungen der Vitamin-C-Hochdosis-Infusionstherapie eine entscheidende Ergänzung zu den Methoden der Schulmedizin darstellen und damit im Rahmen eines gesamtonkologischen Behandlungkonzeptes einen hohen Stellenwert besitzen.

\section{Literatur}

Braschoß A. et al. (Publikation in Vorbereitung); Wesentliche Teile in Sonderdruck «Der Onkologe, Band 12, Heft 1, Januar 2006, Springer Medizin Verlag, Heidelberg, 2006».

Weitere Informationen bei

Pascoe pharmazeutische Präparate $\mathrm{GmbH}$ Schiffenberger Weg 55

35394 Giessen

Tel. +49 641 7960-0, Fax -123

info@pascoe.de

\section{Ticker+++ Ticker+++ Ticker+++ Ticker+++ Ticker+++ Ticker+++ Ticker+++}

biosyn Arzneimittel GmbH. FACTOR AF 2 ist einer der Immunmodulatoren, welcher für Krebspatienten gleich mehrere positive Eigenschaften hat und insgesamt zu einer Verbesserung der Lebensqualität führt. Das in Injektionsampullen angebotene Präparat enthält einen Leber-Milz-Extrakt und wird von der biosyn Arzneimittel $\mathrm{GmbH}$ vermarktet.

Weitere Informationen bei biosyn Arzneimittel GmbH

Pressestelle

Schorndorfer Straße 32, 70734 Fellbach

Tel. +49 711575 32-35

presse@biosyn.de
Steigerwald Arzneimittelwerk GmbH. Mit dem modernen Johanniskraut-Präparat Laif ${ }^{\circledR} 900$ des Unternehmens Steigerwald ist es gelungen, die optimale Dosierung von 900 mg des wertvollen Johanniskrautextraktes in nur eine einzige Tablette zu packen. Laif 900 wird zur Behandlung von depressiven Verstimmungszuständen empfohlen und ist trotz der hohen Dosierung sehr gut verträglich.

Weitere Informationen bei

Steigerwald Arzneimittelwerk GmbH

Havelstraße 5, 64295 Darmstadt

Tel. +49 $61513305-0$
Harras Pharma Curarina Arzneimittel GmbH. Die Ergebnisse einer randomisierten, Placebokontrollierten Doppelblind-Studie zeigen: Sensicutan ${ }^{\circledR}$ cortisonfrei ist ein sehr gut verträgliches und wirksames Therapiekonzept bei Neurodermitis und allergisch bedingten Hautekzemen zur cortisonfreien Akutbehandlung oder intermittierenden Langzeitbehandlung.

Weitere Informationen bei

Harras Pharma Curarina

Arzneimittel $\mathrm{GmbH}$

Ruth Komorek

Am Harras 15, 81373 München

Tel. +49 89747 367-41 